\title{
Erroneous perception of a clinical sign: Sepsis-induced cholestasis mimicking hepatitis in an adolescent patient
}

\author{
Fabrizio Romano ${ }^{1}$, Marisa Molinaro ${ }^{1}$, Susanne Schibli ${ }^{1}$, Philipp Agyemann ${ }^{1}$, and Ruth \\ Löllgen $^{2}$ \\ ${ }^{1}$ Inselspital University Hospital Bern \\ ${ }^{2}$ Karolinska Universitetssjukhuset
}

May 8, 2021

\begin{abstract}
Background: In children and adolescents, isolated cholestasis rarely is a first clinical sign of severe sepsis. Case: A previously healthy 14-year-old adolescent had severe S. aureus sepsis. Delayed diagnosis required a prolonged antimicrobial treatment. Conclusion: This unique case illustrates the importance of considering jaundice as an early sign of sepsis.
\end{abstract}

\section{Hosted file}

CCR_Cholestasis preceding sepsis manuscript.pdf available at https://authorea.com/ users/412547/articles/521220-erroneous-perception-of-a-clinical-sign-sepsis-inducedcholestasis-mimicking-hepatitis-in-an-adolescent-patient

\section{Hosted file}

Figure 1 Cholestasis preceding sepsis case report.pdf available at https://authorea.com/ users/412547/articles/521220-erroneous-perception-of-a-clinical-sign-sepsis-inducedcholestasis-mimicking-hepatitis-in-an-adolescent-patient

\section{Hosted file}

Figure 2 Cholestasis preceding sepsis case report.pdf available at https://authorea.com/ users/412547/articles/521220-erroneous-perception-of-a-clinical-sign-sepsis-inducedcholestasis-mimicking-hepatitis-in-an-adolescent-patient 